\title{
Molecular Evolution of the Vertebrate FK506 Binding Protein 25
}

\author{
Fei Liu, ${ }^{1,2}$ Xiao-Long Wei, ${ }^{3}$ Hao Li, ${ }^{1,2}$ Ji-Fu Wei, $^{2}$ Yong-Qing Wang, ${ }^{2}$ and Xiao-Jian Gong ${ }^{1}$ \\ ${ }^{1}$ Department of Pharmacology, China Pharmaceutical University, Nanjing 210009, China \\ ${ }^{2}$ Research Division of Clinical Pharmacology, The First Affiliated Hospital, Nanjing Medical University, 300 Guangzhou Road, \\ Nanjing 210029, China \\ ${ }^{3}$ Department of Pathology, Cancer Hospital of Shantou University Medical College, Shantou, China
}

Correspondence should be addressed to Yong-Qing Wang; wyqjsh@hotmail.com and Xiao-Jian Gong; gongxj66@sina.com

Received 25 November 2013; Accepted 16 January 2014; Published 2 March 2014

Academic Editor: Huai-Rong Luo

Copyright $\odot 2014$ Fei Liu et al. This is an open access article distributed under the Creative Commons Attribution License, which permits unrestricted use, distribution, and reproduction in any medium, provided the original work is properly cited.

FK506 binding proteins (FKBPs) belong to immunophilins with peptidyl-prolyl isomerases (PPIases) activity. FKBP25 (also known as FKBP3) is one of the nuclear DNA-binding proteins in the FKBPs family, which plays an important role in regulating transcription and chromatin structure. The calculation of nonsynonymous and synonymous substitution rates suggested that FKBP25 undergoes purifying selection throughout the whole vertebrate evolution. Moreover, the result of site-specific tests showed that no sites were detected under positive selection. Only one PPIase domain was detected by searching FKBP25 sequences at Pfam and SMART domain databases. Mammalian FKBP25 possess exon-intron conservation, although conservation in the whole vertebrate lineage is incomplete. The result of this study suggests that the purifying selection triggers FKBP25 evolutionary history, which allows us to discover the complete role of the PPIase domain in the interaction between FKBP25 and nuclear proteins. Moreover, intron alterations during FKBP25 evolution that regulate gene splicing may be involved in the purifying selection.

\section{Introduction}

Immunophilins include three families with peptidyl-prolyl isomerases (PPIases) activity, FK506 binding proteins (FKBPs), cyclophilins, and parvulins. FKBPs are named for binding to the immunosuppressive drug FK506, characterized by one or more PPIase domains. The 15 identified members of human FKBPs are divided into 4 groups: cytoplasmic, TPR domain, endoplasmic reticulum (ER), and nucleus. FKBP25 and FKBP133 locate in the nucleus, containing a single PPIase domain [1].

FKBP25 (also known as FKBP3) is the first mammalian FKBP with a calculated molecular mass of $25 \mathrm{kDa}$ found in the nucleus, which plays a role in regulating transcription and chromatin structure. The FKBP25 comprises a conserved PPIase domain at its $\mathrm{C}$-terminus with a $43 \%$ sequence identity to FKBP12 and a helix-loop-helix (HLH) motif at its unique hydrophilic $\mathrm{N}$-terminal $[2,3]$. This conserved PPIase domain functions in binding to the immunosuppressive agent FK506 or rapamycin. Unlike another FKBPs, FKBP25 shows a strong affinity for binding rapamycin $(\mathrm{Ki}=0.9 \mathrm{nM})$ over
FK506 $(\mathrm{Ki}=200 \mathrm{nM})$ [4]. The FKBP25 was reported to be associated with nuclear proteins including transcription factor Yin-Yang1 (YY1), mouse double minute 2 (MDM2), and histone deacetylases (HDACs) [5]. FKBP25 binds to YY1 at $\mathrm{N}$-terminal and increases its DNA-binding activity without the involvement of the FK506/rapamycin binding domain [6]. In addition, the level and activity of the tumor suppressor protein p53 are negatively regulated by MDM2. The HLH motif of FKBP25 mediates protein-protein interaction to enhance ubiquitination and degradation of oncogene MDM2, increasing the expression of tumor suppressor p53 and its downstream effector p21 [7]. Moreover, the proteinprotein interaction contributes to form HDAC complexes, which is critical for the chromatin structure [2].

In 1992, Jin et al. reported the molecule cloning of human FKBP25 and performed a homology comparison between FKBP25 and FKBP12/FKBP13 [8]. Furthermore, Mas et al. showed the molecule cloning of mouse FKBP25 and expression pattern of FKBP25 gene during cerebral cortical neurogenesis [9]. However, the relationships between nuclear functions and evolution in FKBP25 are seldom reported. 
TABLE 1: Site-specific tests for positive selectionof FKBP25.

\begin{tabular}{|c|c|c|c|c|c|}
\hline Species & Models & Estimates of parameters & $\ln L$ & $2 \Delta l$ & Positively selected sites \\
\hline \multirow[b]{2}{*}{ Vertebrate } & M7 & $p=0.91900 q=8.19764$ & -5463.938465 & \multirow[b]{2}{*}{0.003806} & NA \\
\hline & M8 & $\begin{aligned} p 0= & 0.99999 p=0.91899 q=8.19758 \\
& (p 1=0.00001) w=1.86072\end{aligned}$ & -5463.940368 & & None \\
\hline \multirow[b]{2}{*}{ Mammalian } & M7 & $p=0.33823 q=1.62046$ & -2182.244789 & \multirow[b]{2}{*}{0.000258} & NA \\
\hline & M8 & $\begin{array}{c}p 0=0.99999 p=0.33824 q=1.62055 \\
(p 1=0.00001) w=1.00000\end{array}$ & -2182.244918 & & None \\
\hline \multirow[b]{2}{*}{ Primate } & M7 & $p=4.13016 q=99.00000$ & -997.077389 & \multirow[b]{2}{*}{0.000102} & NA \\
\hline & M8 & $\begin{array}{c}p 0=0.99999 p=4.12942 q=99.00000 \\
(p 1=0.00001) w=1.00000\end{array}$ & -997.077440 & & None \\
\hline \multirow[b]{2}{*}{ Mammalian excluding primate } & M7 & $p=0.28229 q=1.41420$ & -2242.306222 & \multirow[b]{2}{*}{0.000160} & NA \\
\hline & M8 & $\begin{array}{c}p 0=0.99999 p=0.28230 q=1.41430 \\
(p 1=0.00001) w=1.00000\end{array}$ & -2242.306302 & & NS \\
\hline \multirow[b]{2}{*}{ Rodent } & M7 & $p=0.13287 q=1.19752$ & -1372.902164 & \multirow[b]{2}{*}{0.000058} & NA \\
\hline & M8 & $\begin{array}{c}p 0=0.99999 p=0.13287 q=1.19764 \\
(p 1=0.00001) w=1.00000\end{array}$ & -1372.902193 & & NS \\
\hline \multirow[b]{2}{*}{ Teleost } & M7 & $p=0.38691 q=4.30540$ & -2354.923181 & \multirow[b]{2}{*}{0.000408} & NA \\
\hline & M8 & $\begin{aligned} p 0= & 0.99999 p=0.38690 q=4.30545 \\
& (p 1=0.00001) w=3.90806\end{aligned}$ & -2354.923385 & & NS \\
\hline
\end{tabular}

lnL: the log-likelihood difference between the two models; $2 \Delta l$ : twice the log-likelihood difference between the two models (In all the species, $2 \Delta l<9.21$, the $P$-value is more than the significance level 0.05 , indicating that M8 model is not better than M7 model); NA: not allowed; NS: not shown (it means the sites under positive selection but not reaching the significance level of 0.9 ).

In this study, we exhibit an evolutional analysis not only on selective pressure but also on intron-exon conversion among vertebrate FKBP25 genes.

\section{Materials and Methods}

2.1. Sequence Data Collection. All the FKBP25 gene and amino acid sequences were obtained from the ENSEMBL (http://www.ensembl.org/index.html) [10], based on orthologous and paralogous relationships. The gained FKBP25 sequences were applied as queries to search known FKBP25 genes using BLAST at the National Center for Biotechnology Information (NCBI), in order to confirm whether their best hit was an FKBP25 gene [11].

Incomplete sequences of FKBP25 genes in four species (tree shrew, horse, platypus, and turkey) were retrieved from both ENSEMBL and NCBI. After eliminating these incomplete sequences, 28 sequences were applied for this study. The 28 sequences from 23 species comprised human (ENSG00000100442), chimpanzee (ENSPTRG000000063 05), gorilla (ENSGGOG00000013322), orangutan (ENSPP YG00000005778), macaque (ENSMMUG00000016512), marmoset (ENSCJAG00000015972), mouse (ENSMUSG00 000020949), rat (ENSRNOG00000004629), guinea pig (ENS CPOG00000001444), rabbit1 (ENSOCUG00000007535), rabbit2 (ENSOCUG00000026892), dog1 (ENSCAFG00000 014018), $\operatorname{dog} 2$ (ENSCAFG00000014093), $\operatorname{dog} 3$ (ENSCA FG00000024192), dog4 (ENSCAFG00000000 578), cow (ENSBTAG00000002610), elephant1 (ENSLAFG000000035 72), elephant2 (ENSLAFG00000027553), opossum (ENS MODG00000007352), chicken (ENSGALG00000012466), zebra finch (ENSTGUG00000013231), anole lizard (ENS ACAG00000004080), xenopus (ENSXETG00000003052), fugu (ENSTRUG00000011887), medaka (ENSORLG00000 015070), stickleback (ENSGACG00000012834), tetraodon (ENSTNIG00000010980), and zebrafish (ENSDARG00000 079018).

2.2. Molecular Phylogenetic Analyses. The protein coding sequences of FKBP25 were aligned using CLUSTAL W program in MEGA 5.05. We constructed a maximum likelihood (ML) tree of FKBP25 amino acid sequences by MEGA 5.05 with the optimal model (Kimura 2-parameter model). The relative support of internal node was performed by bootstrap analyses with 1000 replications for ML reconstructions [12].

2.3. Selection Pressure Analyses. The numbers of nonsynonymous substitutions per nonsynonymous site $(d N)$ and the numbers of synonymous substitutions per synonymous site $(d S)$ were computed by MEGA 5.05 with the modified NeiGojobori method. The $d N / d S<1,=1$ and $>1$ demonstrate purifying selection, neutral selection, and positive selection, respectively [13]. The $d N$ is the numbers of nonsynonymous substitutions per nonsynonymous site, and the $d S$ is the numbers of synonymous substitutions per synonymous site. The transition/transversion ratio was 1.55 estimated using the ML method by MEGA 5.05 [14].

The FASTA format of FKBP25 sequences was converted to the PAML format using DAMBE software for subsequent site analyses [13]. The CODEML program implemented in the PAML 4.7 package was used to detect positive selection of individual sites. The site-specific model was exerted using 


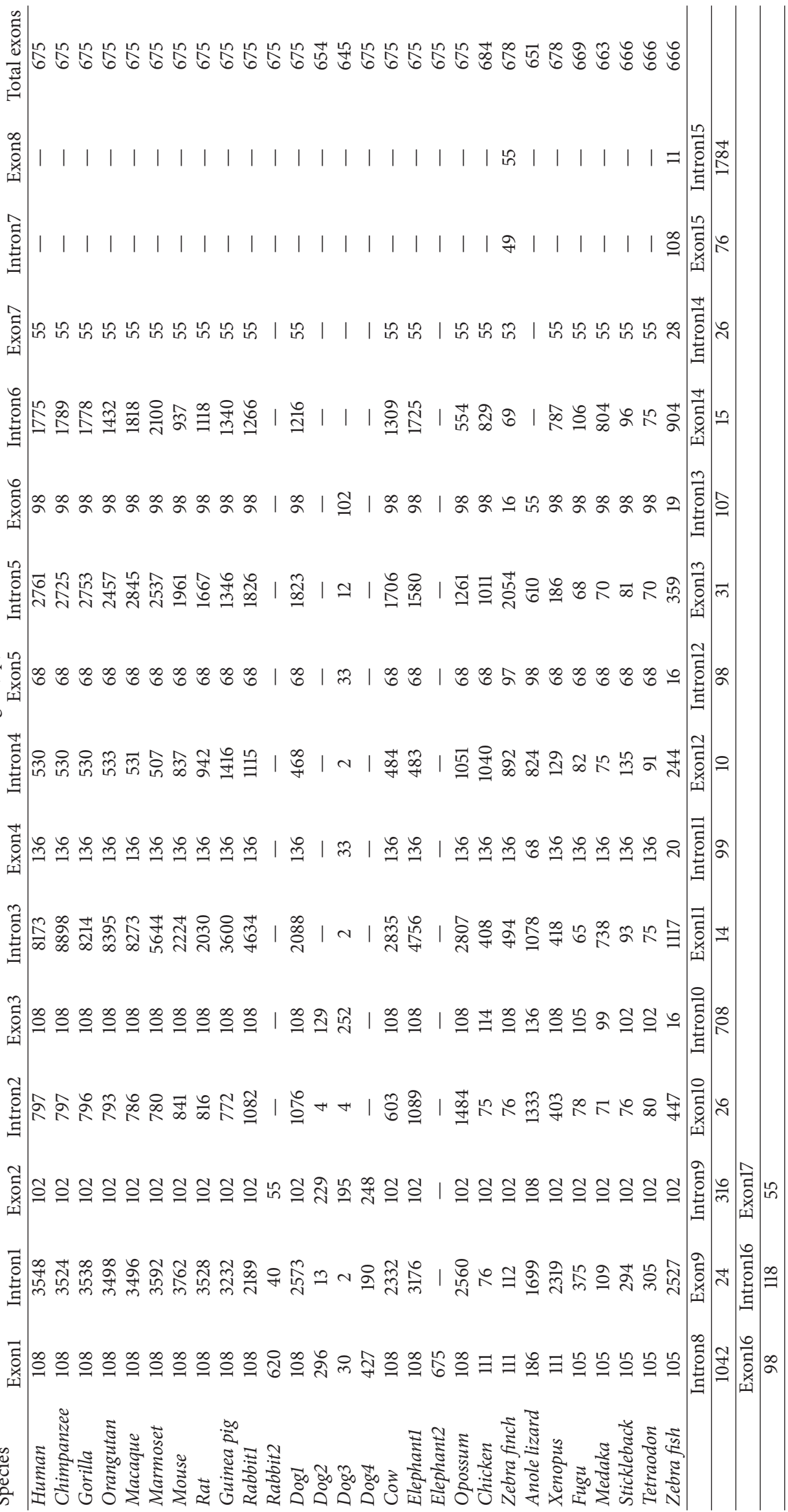




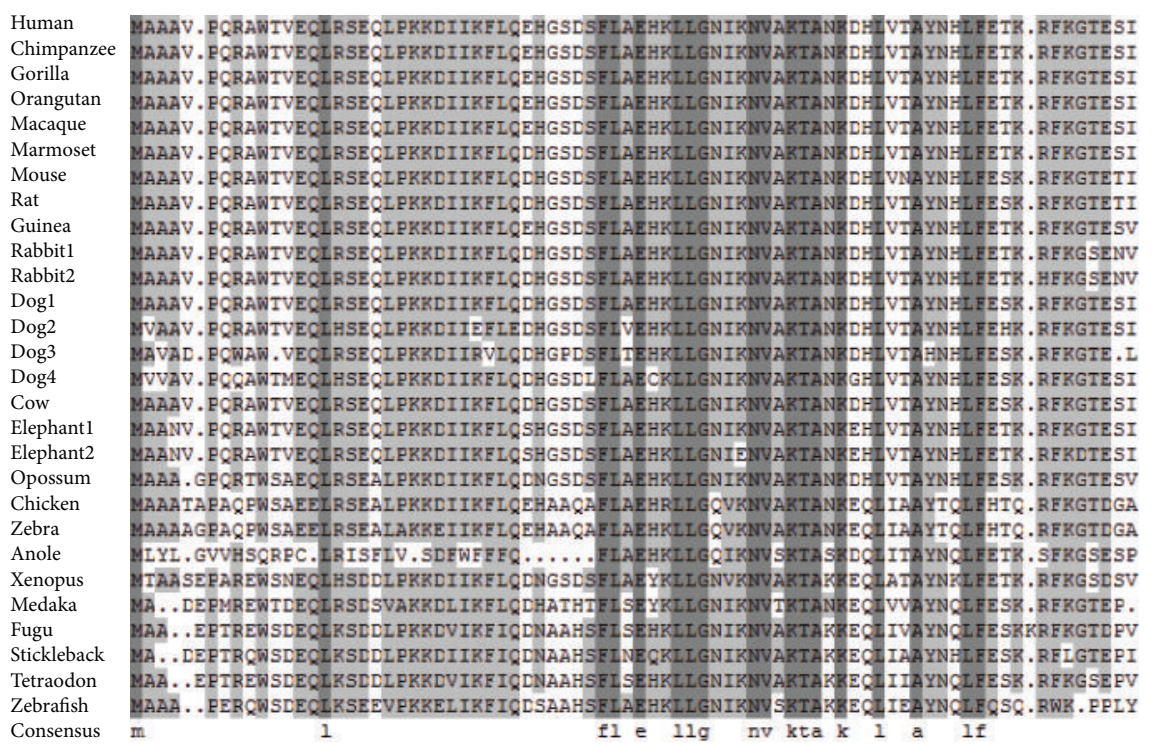

Human

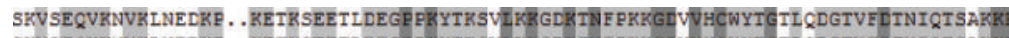

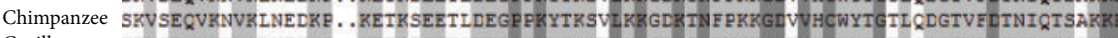

Gorilla SKVSECVRNVRLNEDKP. . KETRSEETLDEGPPKYTRSVLKRGDKTNFPKRGIVVHCWYTGIL CDGTVFDTNIQTSAKRI

Orangutan SKV SECVRNVRLNEDKP. . RETRSEETLDEGPPRYTRSVIRR GDKTNF PKKGLVVHCWYT IL CDGTVFDTNICISAKK

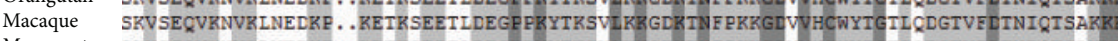

Marmoset SKVSEQVRNVRLNEDRP. . RETRSEETLDEGPPRYTRSVLKRGDRTNE PRKGIVVHCWYTGTL CDGTVFLTNI

Mouse SKVSECVRNVRLSDDKR...RLSRSEETLDEGPPRYTRS ILKRGDKTNF PKRGLVVHCWYTGIIPDGTVFLTNIQTSSKR

Rat SKVSEQVRNVRLNDDRC...RLSRSEETLDEGPRKYTKSVLKRGDKTNFPKKGLVVHCWYTGII PDGTVEDTNIQTSSK

Guinea SKVTDQVRNVRLNEEKF...KETKSEETQDEGERKYTKSIIKRGDKTNF PKKGLVVHCWYTGII CDGTVFLTNIQTSAKY

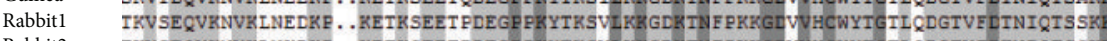

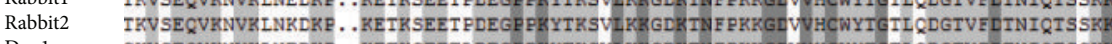

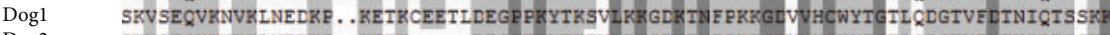

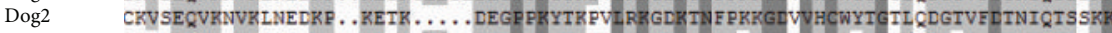

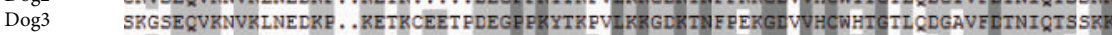

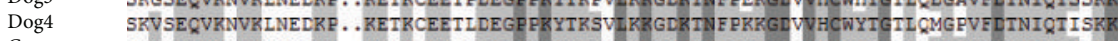

Cow SKVSEQVRNVRLNEDKF. . KETRSEETLDEGP PRYTRSV KRGDKTNF PKKGDVVHCWYT TI CDGTVFDTNIQTS SKR

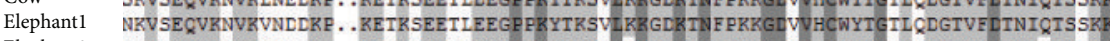

Elephant2 NNVLECQVRNVRVNDDKF...RETRSEETLEEGPFKYTKSVL QRGDKTNF PKKGLVVHCWYT II CDGTVFLTNIPISSKK

Opossum AKVTEQVRSVRLDEERS. . KEVRPEETLDEGPPKYTRS ILKR GDKTNF PKKGLVVHCWYT GIL QDGTVFDTNIQTSAKR

Chicken ERAAEKARPGKAEGEKEKDKAAKAEEPAEEGPRYTKS I KKGDKTNF PKKGITHCWYTGKI QDGTVFLTNVQISSKL

Zebra EKAAERARPARAEEARG. . RAVRAEEAVEEGPPKYTKS I KRGDKTNF PKKGLIVHCWYT GRI CDGTVFDINIC्रSSSKK

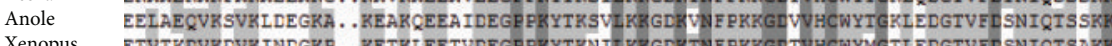

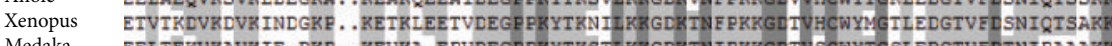

Medaka EEITEKVRAVRIE.DKP. . KEVRA. EPVDEGPPKYTRSILKRGDKTNIPKKGDIV SCWYT GSIEDGTVFDTNIPAAAKKI

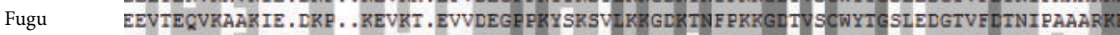

Stickleback EEVTECVRSVRLE. ERP...RDVPA. EVVDEGPPRYFRSVIKRGDRENEPRKGINVSCWYTGSIEDGTVFDTNIPTTARRM

Tetraodon EEVTEQVRAARIE. EKE. . REVRT.EAVDEGPPRYTRSVLKRGDKTN F PRKGITV SCWYTGSLEDGTVFLTNIPTAARKI

Zebrafish
Consensus

156

Human

RNARFLSERVGVGKVIRGWDEALLTMSRGERARLEIEPEWAYGKRGCEDAKIPPNAKIIFEVELVDID

Chimpanzee RNARPLSFRVGVGKV IRGWDEALITMSKGEKARLEIEPEWAYGKKGQPDAKIPPNAKI IFEVELVDID

Gorilla RNARPLSFRVGVGKVIRGWDEALITMSRGERARLEIE PEWAYGKKG QPDAKIPPNAKI IFEVELVDID

Orangutan RNARPLSERVGVGKVIRGWDEALLTMSKGERARLEIEPEWAYGKKGQPDAKI PPNAKL IFEVELVDID

Macaque RNARPLSFRVGVGKVIRGWDEALITMSRGEKARLEIEPEWAYGKRGQPDAKIP PNAKI IFEVELVDID

Marmoset RNARPLSERVGVGKVIRGWDEALLTMSRGERARLE IEPEWAYGKRGQPDAKI P PNAKI IFEVELVDID

Mouse RNARPLSERVGVGKVIRGWDEALITMSRGRRARLEIEPEWAYGRRGCPDARIPPNTKI TFEVELVDID

Rat

Guinea

Rabbit1

Dogl

Dog2

Dog2

Dog3

$\operatorname{Dog} 4$

Elew

Elephant2

Opossum

Chicken

Zebra

Anole

Xenopus
Medaka

Fugu

Stickleback

Tetraodon

Zebrafish NIP 作 KNAKPLSFKVGVGKV IRGWDEALITMSRGEKARLE IEPEWAYGKKGQPDAKIP PNAKI IFEVELVDID RNAKPLSFKVGVGKV IRGWDEALITMSKGEKARLEIEPEWAYGKKGQEDAKIPENAKL IFEVELVDID RNAKPLSFKVGVGKV IRGWDEALITMSRGEKALLEIEPEWAYGKRGQEDAKIPPNAKI IFEVELVDID KNARPLSERVGIGKV IRGWDEALITMSRGEKARLEIEPEWAYGKKGQ PDAKIPFNAKI IFEVELVDID .NAKPLSFRAG IDKV IRGWDEALIT.SRGERARLEIEPEWAYGKRGCEDAKIPPNGKI IFEVELVDID

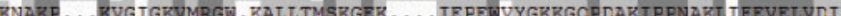

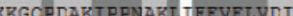
NARELSTVEIGKVIRGWDEALITMSKGEKARLEIEPEWAYGKKGQPDAKIPFNAKI IFEVELVDID RNARPLSFRVGIGKV IRGWDEALITMSKGERARLEIEPEWAYGKRGQPDAKIP PNAKI IFEVELVDID RNARPLSFRVGVGKV IRGWDEALLTMSKGERARLEIEPEWAYGRRGCPDAKIP PNAKI IFEVELVDID RNARPLSFRVEVGKVIRGWDEALLTMSKGEKACLEIEPEWAYGKKGQPDAKIP PNATI IFEVELVDID RNAR PLSFRVGVGKV IRGWDEALITMSKGERARLE IE PEWAYGKKGQ FDAKI PPNAKL NFEVELVDID RAAKPLSEKVGVGKVIRGWDEALITMSKGEKAOIEIEPDWAYGKKG OPDAKIP ENAKI FFEVEIVDI

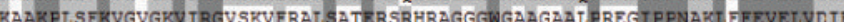
作 RTAKPLSFKVGVGKV IRGWDEALITMSKGEKAHLEIEPEWAYGKKGQPDAKIP PNAKL FFEVELVDIE RAAKELSFRVGVGKV IRGWDEALITMSKGEKAKLEIEPEWAYGRKGI PDAKI P PNAKL FFEVELVDID RQIKPLSFRVGL GRV IRGWDEALITMSKGETARLEIEPEWAYGKRGL PDSKIP PNAKI IFEVELVSVD BQARPLSEKVGLGRVIRGWDEALLTMSRGETARLEIDPEWAYGRKGI PDSKIP NAKIVFEVELVSVD BQTRELT FRVGL GRV IRGWDEG IMTMSKGETSRLE IE PEWAYGRKGL PDSKIP ENAKI IFEVELVAVD RCARPLSFRVGMGRV IRGWDEALITMSKGERARLEIDPEWAYGKRGI PSSK I P PNAKI IFEVELVSVD

Consensus

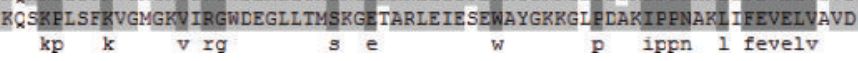

FIGURE 1: Sequence and structural alignment of FKBP25. 


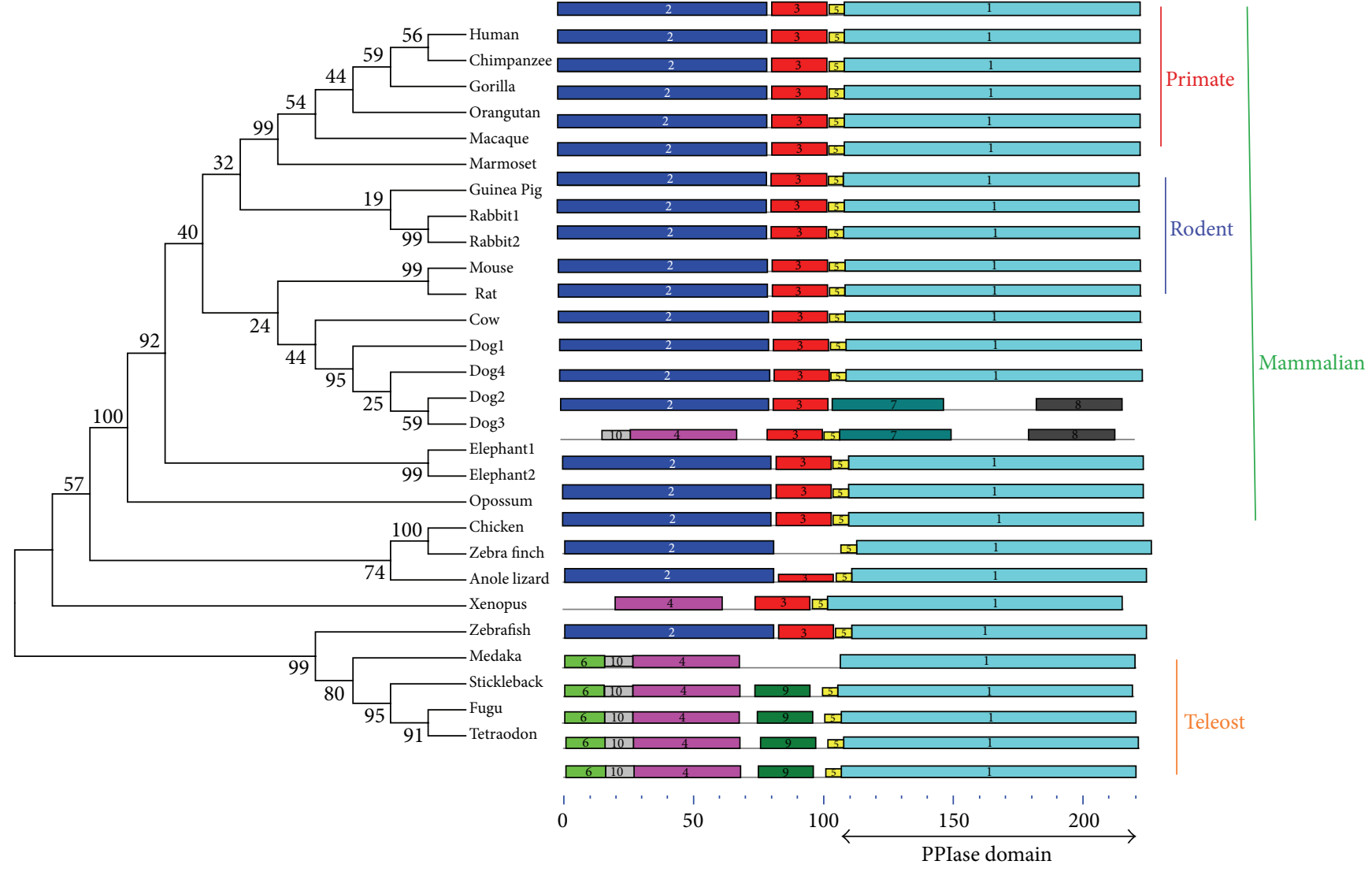

Figure 2: Phylogenetic tree and motif distributions of FKBP25.

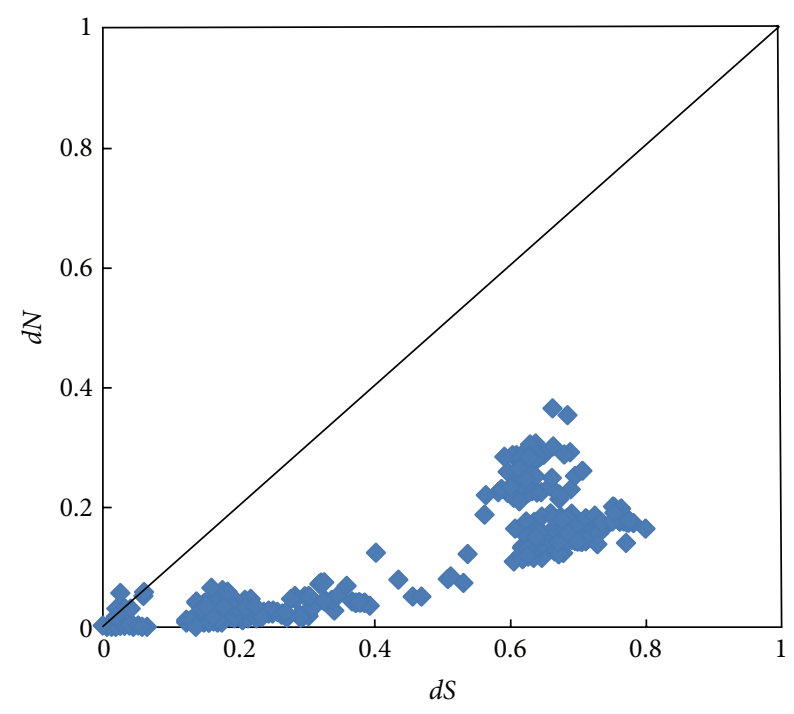

FIGURE 3: Pairwise comparisons of $d N$ and $d S$ among 28 vertebrate FKBP25 sequences.

likelihood ratio tests (LRT) to compare M7 (null model) with M8 model. M7 is a null model that does not allow for any codons with $\omega>1$, whereas M8 model allows for positively selective sites $(\omega>1)$. When the M8 model fitted the data significantly $(P$-value $<0.05)$ better than the null model $(\mathrm{M} 7)$,

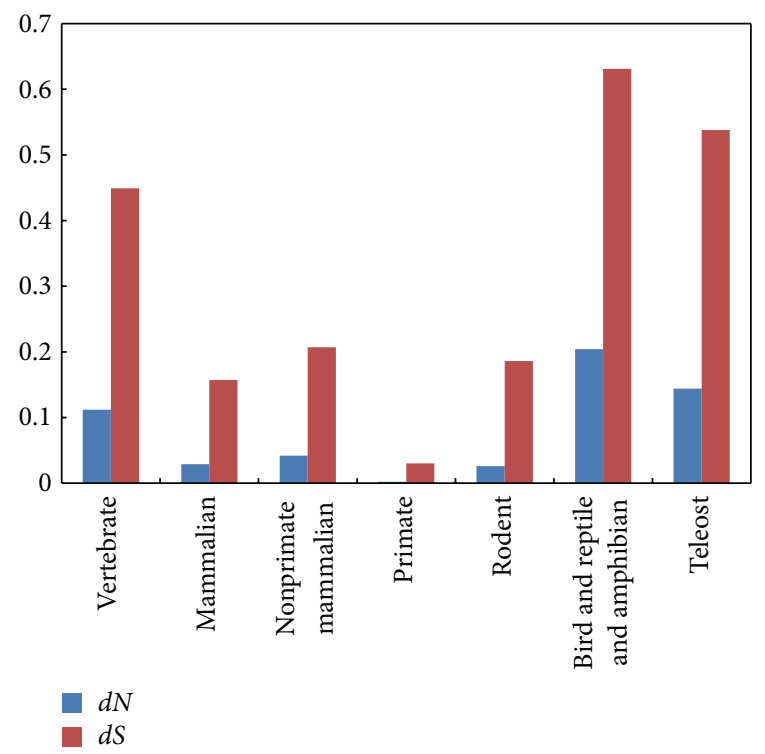

FIGURE 4: The average nonsynonymous $(d N)$ and synonymous $(d S)$ in FKBP25 from different vertebrate groups. The value of average $d N$ was in blue, and the value of average $d S$ was in red.

the presence of sites with $\omega>1$ is suggested. On the contrary, the results of $P$ value $>0.05$ proved the absence of sites with $\omega>1$. The twice log likelihood difference between the two compared models $(2 \Delta l)$ is compared against $\chi^{2}$ with 


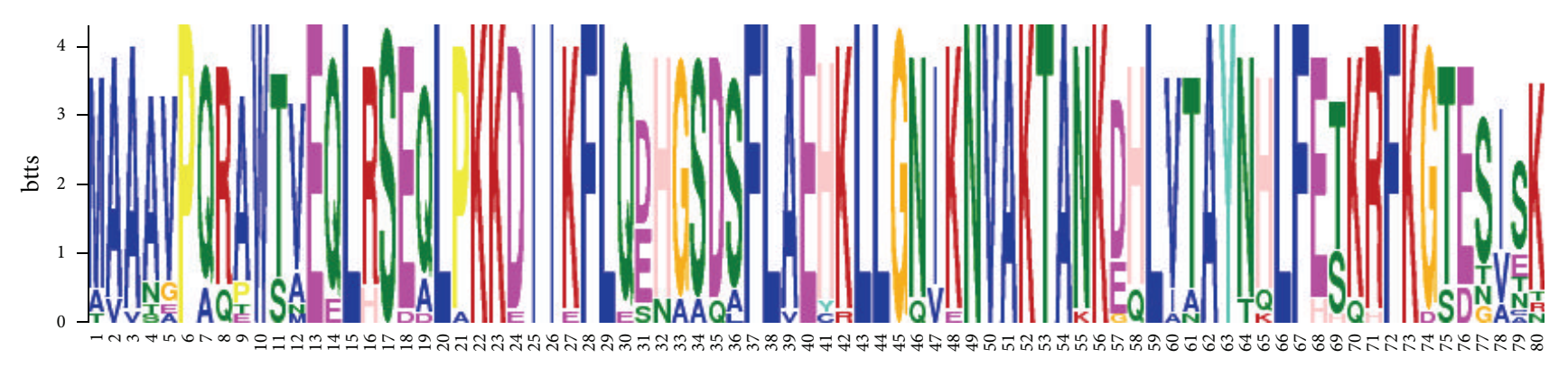

(a)

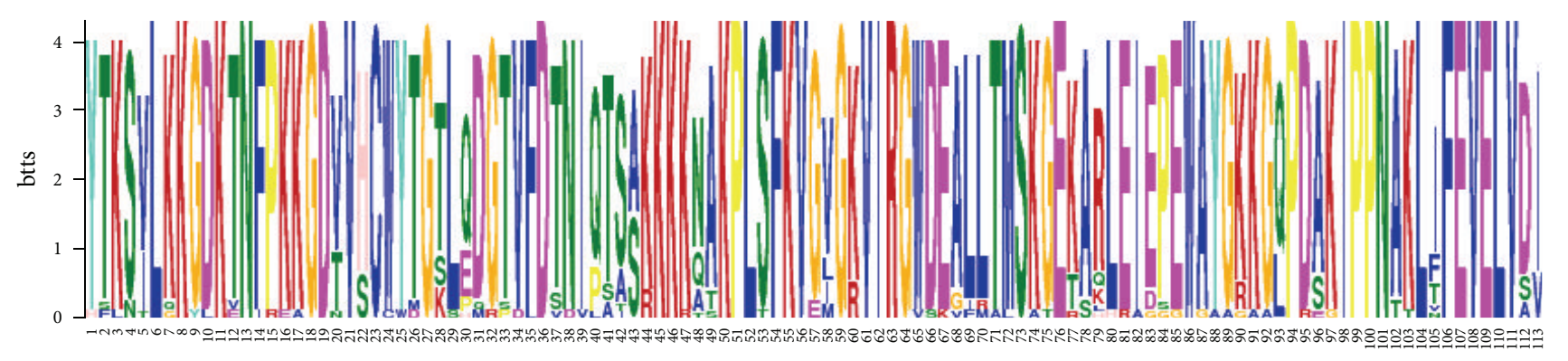

(b)

FIGURE 5: Sequence logos (MEME LOGOs) of conserved motifs identified in vertebrate FKBP25.

critical values 5.99 and 9.21 at 0.05 and 0.01 significance levels, respectively [15].

2.4. Protein Domain and Motif Analyses. Protein domain analyses of FKBP25 were shown at Pfam domains database (http://pfam.sanger.ac.uk) [16]. SMART (http://smart.emblheidelberg.de/) was used to make sure the presence of FKBP25 domains [17]. The motifs of FKBP25 were analyzed by the MEME software (http://meme.sdsc.edu/meme/website/intro.html) with a maximum of 10 motifs to find [18].

2.5. Exon-Intron Conservation Analyses. We collected elaborate information about FKBP25 exon and intron from ENSEMBL (http://www.ensembl.org/index.html) [19]. The number and length of FKBP25 exon and intron in 28 sequences were investigated for exon-intron conservation analyses.

\section{Results}

3.1. Phylogenetic Analyses of FKBP25. All the FKBP25 gene and protein sequences were collected from the ENSEMBL and checked by BLAST at NCBI. The sequence and structural alignment of FKBP25 was shown in Figure 1. The phylogenetic tree was constructed according to the protein coding sequences of FKBP25 using the maximum likelihood method (Figure 2, left panel). The FKBP25 genes from the primate lineage and teleost lineage form a species-specific cluster, respectively. Four FKBP25 isoforms of $d o g$ exhibited a close relationship and clustered together, according to the phylogenetic tree. There were similar phenomena in rabbit and elephant.
3.2. Selection Pressure Analyses. The nonsynonymous to synonymous rate ratio $(d N / d S)$ may demonstrate the selective pressures of involved protein. We calculated the pairwise distance of FKBP25 sequences using MEGA 5.05. There was a significantly lower $d N$ than $d S$ in the pairwise comparisons of these sequences. Most values of $d N / d S$ in these sequences were distributed blow the diagonal, showing that the presence of a purifying selection existed in the FKBP25 (Figure 3). The comparisons of average $d N$ and $d S$ in various vertebrate groups were shown in Figure 4, respectively. Furthermore, site-specific tests were performed for searching the positive selection sites in vertebrate, mammalian, primate, and mammalian excluding primate, rodent and teleost lineages. Although some positive selection sites were computed, each $2 \Delta l$ of M7 and M8 $<5.99$ indicated that the M8 model was not significantly better than the M7 model to fit the data. Consequently, we concluded that the site-specific analyses also compute no positive selection sites acting on FKBP25 using PAML4.7 (Table 1).

3.3. Protein Domain and Motif Analyses. Early studies reported that mammalian FKBP25 have two portions: one is a putative helix-loop-helix motif within $\mathrm{N}$-terminal unique sequence (Figure 5(a)) and the other is the PPIase domain at its C-terminus (Figure 5(b)) [20].

The domain distribution of FKBP25 was investigated using FKBP25 to search amino acid sequences at the Pfam database firstly. Only one domain (PPIase domain) was found in the Pfam database. The PPIase domain within FKBP25 sequences generally started at position 122 and ended at position 221. Similarly, we further make sure that the FKBP25 domain is at SMART, resulting in the single PPIase domain at position 119 to 221 . 


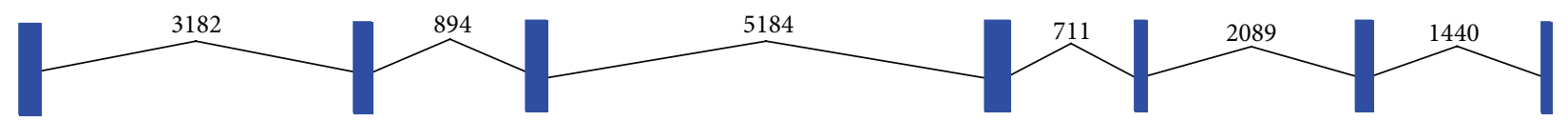

(a) Mammalian

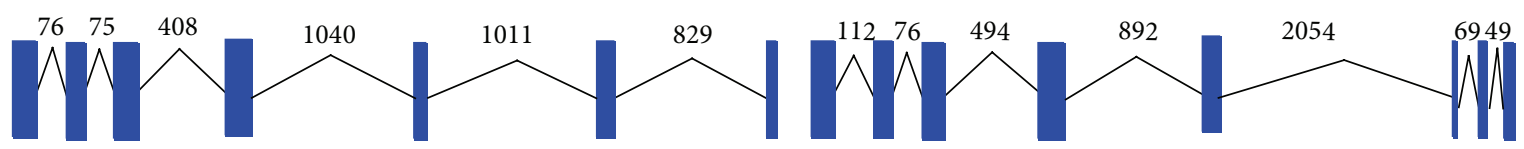

(b) Chicken

(c) Zebra finch

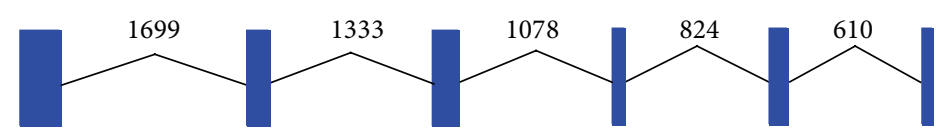

(d) Anole lizard

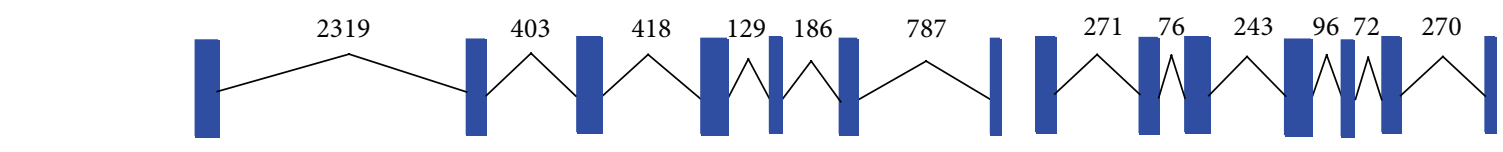

(e) Xenopus

(f) Teleost excluding zebrafish

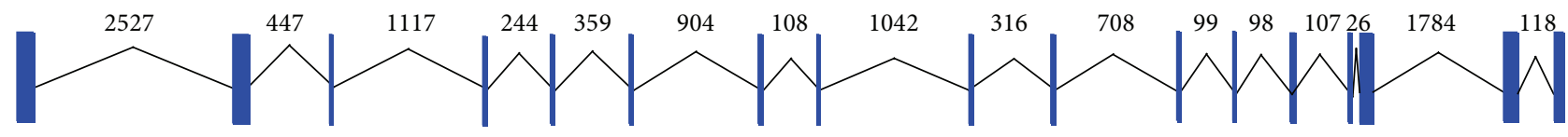

(g) Zebrafish

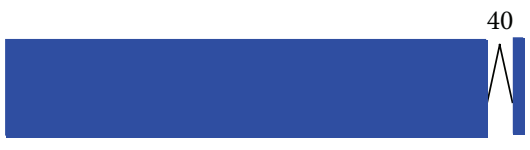

(h) Rabbit2

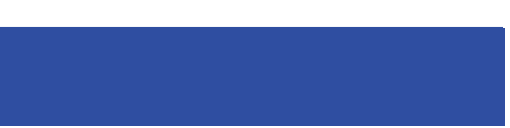

(i) Elephant2

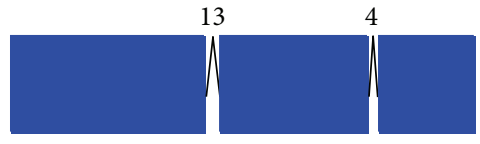

(j) $\operatorname{Dog} 2$

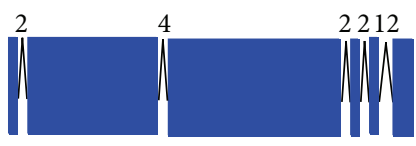

(k) $\operatorname{Dog} 3$

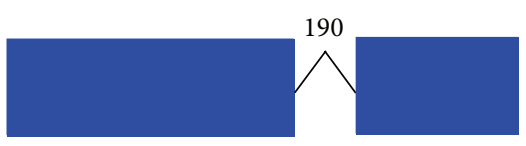

(l) $\operatorname{Dog} 4$

FIGURE 6: Exon-intron conservation among FKBP25 genes.

We then performed a detailed domain and motif analyses using the MEME software. Except two dog isoforms, dog2 and $\operatorname{dog} 3$, the FKBP25 sequences used in this study contain a conversed PPIase domain within motif 1 (shown in Figure 2) at its C-terminus. In addition, the result implied that motif 2 located in the N-terminal contained an HLH motif [6], which was associated with DNA binding and dimerization [21]. However, HLH motif was not found in $\operatorname{dog} 3$, anole lizard, and teleost lineage, implying that these FKBP25 proteins may function on gene expression in another pathway.

3.4. Exon-Intron Conservation Analyses. The exon-intron information collected from the ENSEMBL database was shown in Table 2 and Figure 6. Most of the FKBP25 genes have 7 exons with similar length in different species (Table 2). Mammalian FKBP25 shows exon-intron conservation with 6 introns and similar sizes of each intron. Intron deletions existed in several isoforms of species. The rabbit2 isoform had 2 exons, and elephant 2 isoform had only one exon. The exon numbers of $\operatorname{dog} 2, \operatorname{dog} 3$, and $\operatorname{dog} 4$ isoforms were less than seven. Except mammalian FKBP25 genes, anole lizard reduced one exon compared with mammalian and birds, but the xenopus and teleost maintained 7 exons. The intron deletions of FKBP25 genes may happen in the evolutionary process from amphibian to reptile. Then, a subsequent intron insertion occurred in the evolution from reptile to more advanced animals. The FKBP25 genes also had intron insertion in zebra finch and zebra fish.

\section{Discussion}

FKBP25 is a nuclear member of the FKBPs family that is associated with transcription and chromatin structure [2]. The interactions of FKBP25 with nuclear proteins are closely associated with HLH motif at the N-terminal of FKBP25. However, whether the PPIase domain at C-terminus is important for these interactions remains uncertain. The selection pressure analyses revealed that the purifying selection triggered a whole evolutionary history of FKBP25 in vertebrates, even in each lineage of vertebrates. Purifying selection is one 
of the natural selections that resist deleterious mutations with negative selective coefficients [22]. The mutations that disrupt the correct folding of the FKBP25 domain can weaken PPIase activity and may be the deleterious mutations [5]. It was hypothesized that the mutations of PPIase domain were one of explanations behind the purifying selection throughout FKBP25 evolution. Therefore, although the PPIase domain of FKBP25 was not found to be involved in the protein interactions previously, the PPIase domain might have some associations with the YY1 DNA-binding, MDM2 autoubiquitination and degradation, and HDACs complex formation. These inferences will become a potent direction for exploring the relationship between nuclear proteins and PPIase domain in the future.

The protein-coding sequence length of vertebrate FKBP25 is highly conversed that almost all the taxa are $224 \mathrm{bp}$; nevertheless the original gene length and exonintron status are tremendously various among vertebrate species. However, mammalian FKBP25 exhibit exon-intron conservation with 6 introns and similar sizes of each intron. Chicken FKBP25 maintains 6 introns, but zebra finch has one more intron that inserts in the gene. Similarly, a large variability of intron number and sizes among all the taxa shown in Figure 6 revealed that intron insertion and deletion events happened frequently during the FKBP25 evolutionary history from teleost to birds. In particular, zebrafish demonstrated the maximum number of introns in this study, and the size of exon is much smaller than other teleost species (Figure 6(g)). The intron loss of FKBP25 gene from species more advanced than zebrafish is likely to induce alterations of gene expression due to the absence of specific intron splicing. Under the purifying selection, the FKBP25 gene expression event continuously removes the pernicious mutations that may associate with intron splicing regulation [23].

FKBP25 gene knockdown declined the expression levels of p53 and p21, which emphasized the significance of FKBP25 in regulating p53 and subsequently p21 expression through controlling the ubiquitination of MDM2. Both the FKBP25 PPIase domain and its $\mathrm{N}$-terminal portion were critical for the ubiquitination and degradation of MDM2 [2]. Moreover, Jin et al. reported that FKBP25 prefers to bind to rapamycin rather than FK506, implying that FKBP25 may be an important target molecule for immunosuppression by rapamycin [8]. All the evolution analyses indicated the conservation of FKBP25 gene in vertebrates. Therefore, FKBP25 possesses some basic functions in vertebrate species, like regulating p53 and p21 expression and binding to rapamycin for immunosuppression, reinforcing the suggestion that the purifying selection triggered the evolution of vertebrate FKBP25.

In conclusion, FKBP25 as a nuclear FKBP subjects to the purifying selection throughout the whole evolution, which implied the complete role of the PPIase domain involved in the interaction between FKBP25 and the nuclear proteins that are needed to be discovered continually. Additionally, incomplete exon-intron conservation of FKBP25 meets the vertebrate lineage. The intron gain or loss among the taxa is likely to be involved in the purifying selection.

\section{Conflict of Interests}

The authors declare that there is no conflict of interests regarding the publication of this paper.

\section{Authors' Contribution}

Fei Liu and Xiao-Long Wei contributed to this paper equally.

\section{Acknowledgments}

This project was sponsored by the Grants from the National Natural Science Foundation of China (81273593, 81273274, and 81302331), the Priority Academic Program Development of Jiangsu Higher Education Institutions, National Major Scientific, Technological Special Project for "Significant New Drugs Development” (2011ZX09302-003-02), Jiangsu Province Major Scientific and Technological Special Project (BM2011017), Jiangsu Province's Key Provincial Talents Program (RC201170 and H201108), and the Foundation of the Nanjing Pharmaceutical Association, China (Nanjing, China) (Grant no. H2011YX001).

\section{References}

[1] E. A. Blackburn and M. D. Walkinshaw, "Targeting FKBP isoforms with small-molecule ligands," Current Opinion in Pharmacology, vol. 11, no. 4, pp. 365-371, 2011.

[2] Y.-L. Yao, Y.-C. Liang, H.-H. Huang, and W.-M. Yang, "FKBPs in chromatin modification and cancer," Current Opinion in Pharmacology, vol. 11, no. 4, pp. 301-307, 2011.

[3] S. Riviere, A. Menez, and A. Galat, "On the localization of FKBP25 in T-lymphocytes," FEBS Letters, vol. 315, no. 3, pp. 247251, 1993.

[4] J. Liang, D. T. Hung, S. L. Schreiber, and J. Clardy, "Structure of the human $25 \mathrm{kDa}$ FK506 binding protein complexed with rapamycin," Journal of the American Chemical Society, vol. 118, no. 5, pp. 1231-1232, 1996.

[5] G. Gudavicius, H. Soufari, S. Upadhyay et al., "Resolving the functions of peptidylprolyl isomerases: insights from the mutagenesis of the nuclear FKBP25 enzyme," Biochemical Society Transactions, vol. 41, no. 3, pp. 761-768, 2013.

[6] W.-M. Yang, Y.-L. Yao, and E. Seto, “The Fk506-binding protein 25 functionally associates with histone deacetylases and with transcription factor YY1," EMBO Journal, vol. 20, no. 17, pp. 4814-4825, 2001.

[7] A. M. Ochocka, P. Kampanis, S. Nicol et al., "FKBP25, a novel regulator of the 53 pathway, induces the degradation of MDM2 and activation of p53," FEBS Letters, vol. 583, no. 4, pp. 621-626, 2009.

[8] Y.-J. Jin, S. J. Burakoff, and B. E. Bierer, "Molecular cloning of a $25-\mathrm{kDa}$ high affinity rapamycin binding protein, FKBP25," Journal of Biological Chemistry, vol. 267, no. 16, pp. 10942-10945, 1992.

[9] C. Mas, I. Guimiot-Maloum, F. Guimiot et al., "Molecular cloning and expression pattern of the Fkbp25 gene during cerebral cortical neurogenesis," Gene Expression Patterns, vol. 5, no. 5, pp. 577-585, 2005.

[10] P. Flicek, I. Ahmed, M. R. Amode et al., "Ensembl 2013,” Nucleic Acids Research, vol. 41, no. D1, pp. D48-D55, 2013. 
[11] M. Johnson, I. Zaretskaya, Y. Raytselis, Y. Merezhuk, S. McGinnis, and T. L. Madden, "NCBI BLAST: a better web interface," Nucleic Acids Research, vol. 36, supplement 2, pp. W5-W9, 2008.

[12] S. Kumar, M. Nei, J. Dudley, and K. Tamura, "MEGA: a biologist-centric software for evolutionary analysis of DNA and protein sequences," Briefings in Bioinformatics, vol. 9, no. 4, pp. 299-306, 2008.

[13] X. Xia and Z. Xie, "DAMBE: software package for data analysis in molecular biology and evolution," Journal of Heredity, vol. 92, no. 4, pp. 371-373, 2001.

[14] K. Tamura, D. Peterson, N. Peterson, G. Stecher, M. Nei, and S. Kumar, "MEGA5: molecular evolutionary genetics analysis using maximum likelihood, evolutionary distance, and maximum parsimony methods," Molecular Biology and Evolution, vol. 28, no. 10, pp. 2731-2739, 2011.

[15] Z. Yang, "PAML 4: phylogenetic analysis by maximum likelihood," Molecular Biology and Evolution, vol. 24, no. 8, pp. 15861591, 2007.

[16] R. D. Finn, J. Tate, J. Mistry et al., "The Pfam protein families database," Nucleic Acids Research, vol. 36, no. 1, pp. D281-D288, 2008.

[17] I. Letunic, T. Doerks, and P. Bork, "SMART 7: recent updates to the protein domain annotation resource," Nucleic Acids Research, vol. 40, no. D1, pp. D302-D305, 2012.

[18] T. L. Bailey and C. Elkan, "Fitting a mixture model by expectation maximization to discover motifs in biopolymers," in Proceedings of the International Conference on Intelligent Systems for Molecular Biology, vol. 2, pp. 28-36, 1994.

[19] T. Hubbard, D. Barker, E. Birney et al., "The Ensembl genome database project," Nucleic Acids Research, vol. 30, no. 1, pp. 3841, 2002.

[20] M. Leclercq, F. Vinci, and A. Galat, "Mammalian FKBP-25 and its associated proteins," Archives of Biochemistry and Biophysics, vol. 380, no. 1, pp. 20-28, 2000.

[21] W. D. Kohn, C. T. Mant, and R. S. Hodges, “ $\alpha$-helical protein assembly motifs," Journal of Biological Chemistry, vol. 272, no. 5, pp. 2583-2586, 1997.

[22] Z. Yang and J. R. Bielawski, "Statistical methods for detecting molecular adaptation," Trends in Ecology and Evolution, vol. 15, no. 12, pp. 496-503, 2000.

[23] A. Resch, Y. Xing, A. Alekseyenko, B. Modrek, and C. Lee, "Evidence for a subpopulation of conserved alternative splicing events under selection pressure for protein reading frame preservation," Nucleic Acids Research, vol. 32, no. 4, pp. 12611269, 2004. 

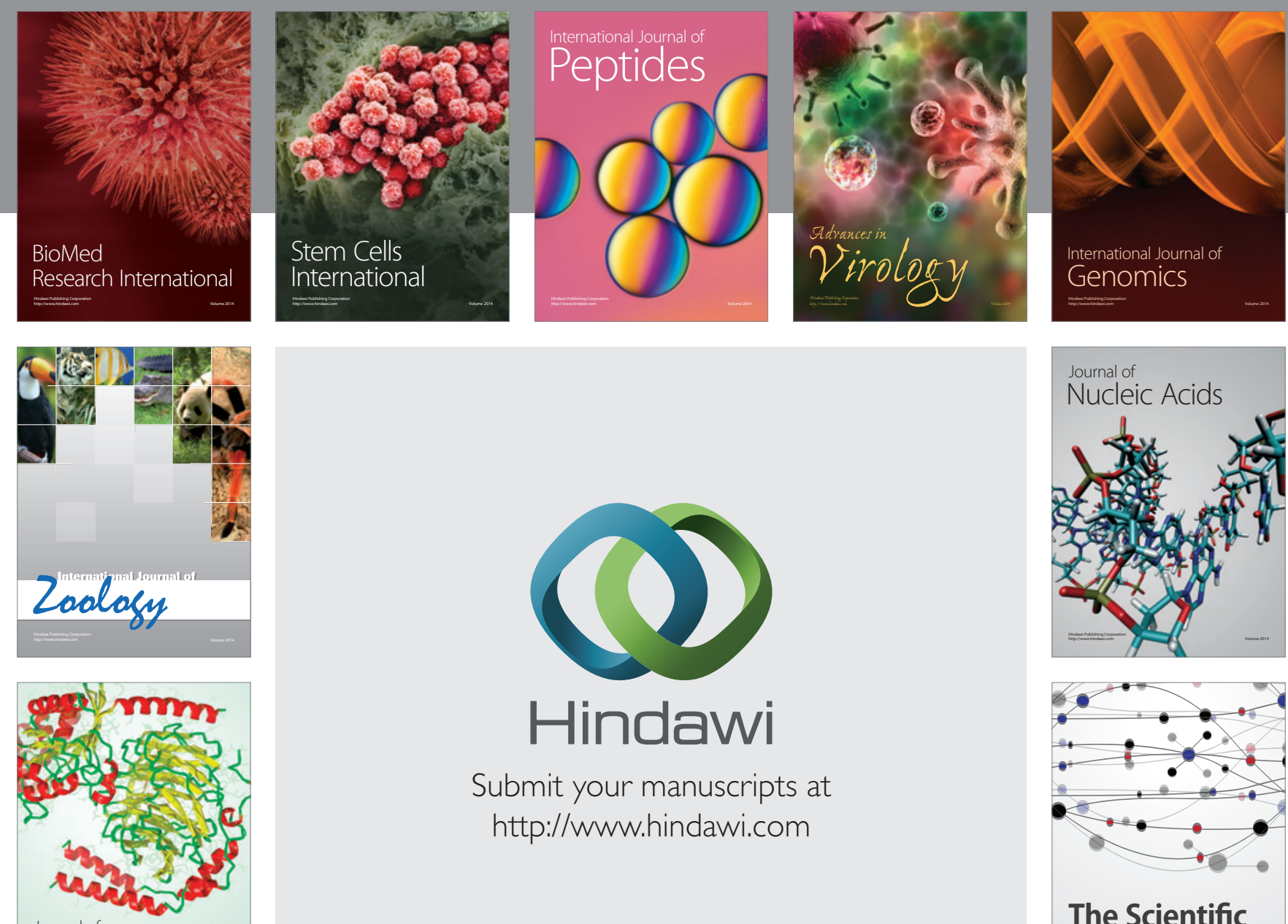

Submit your manuscripts at

http://www.hindawi.com

Journal of
Signal Transduction
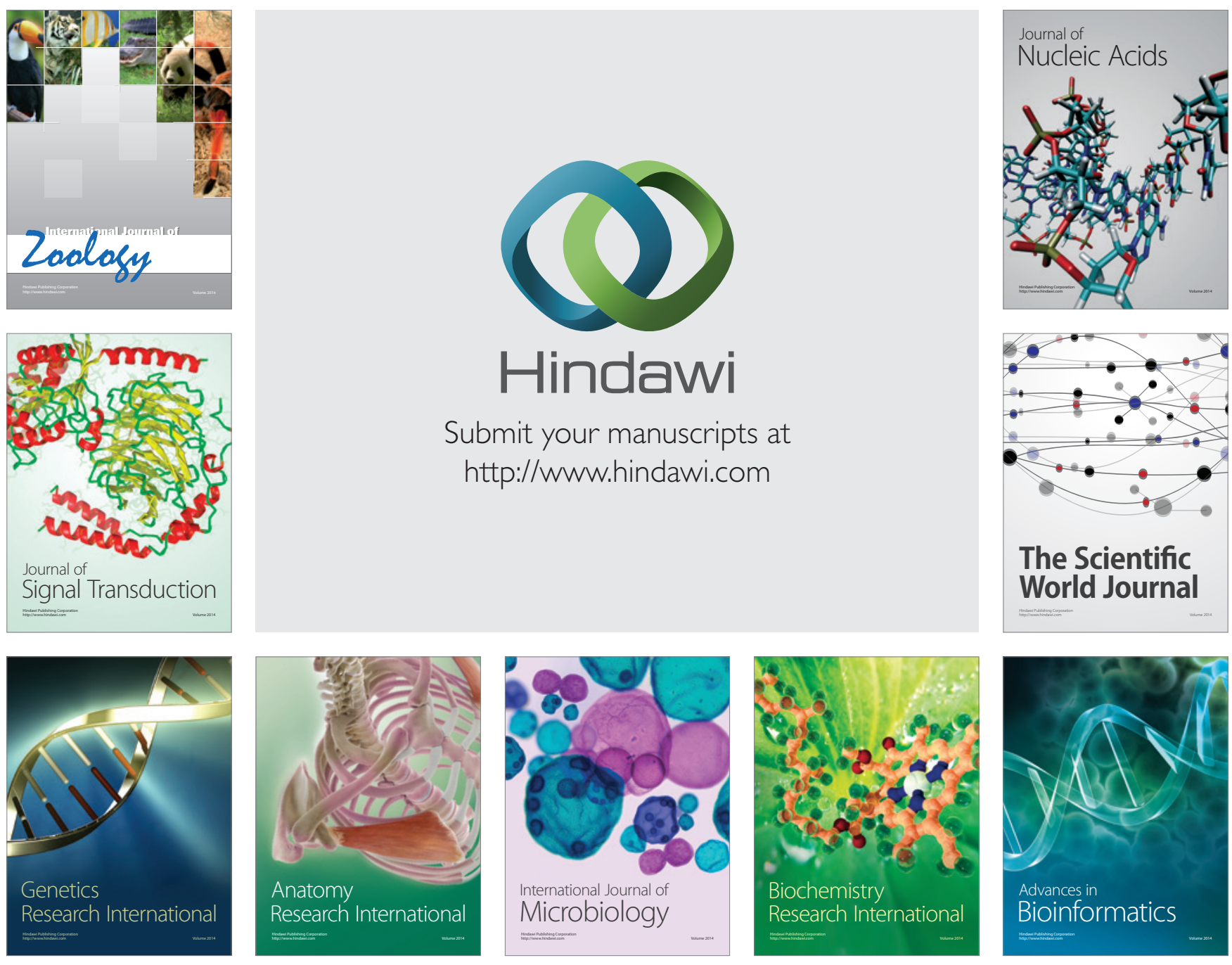

The Scientific World Journal
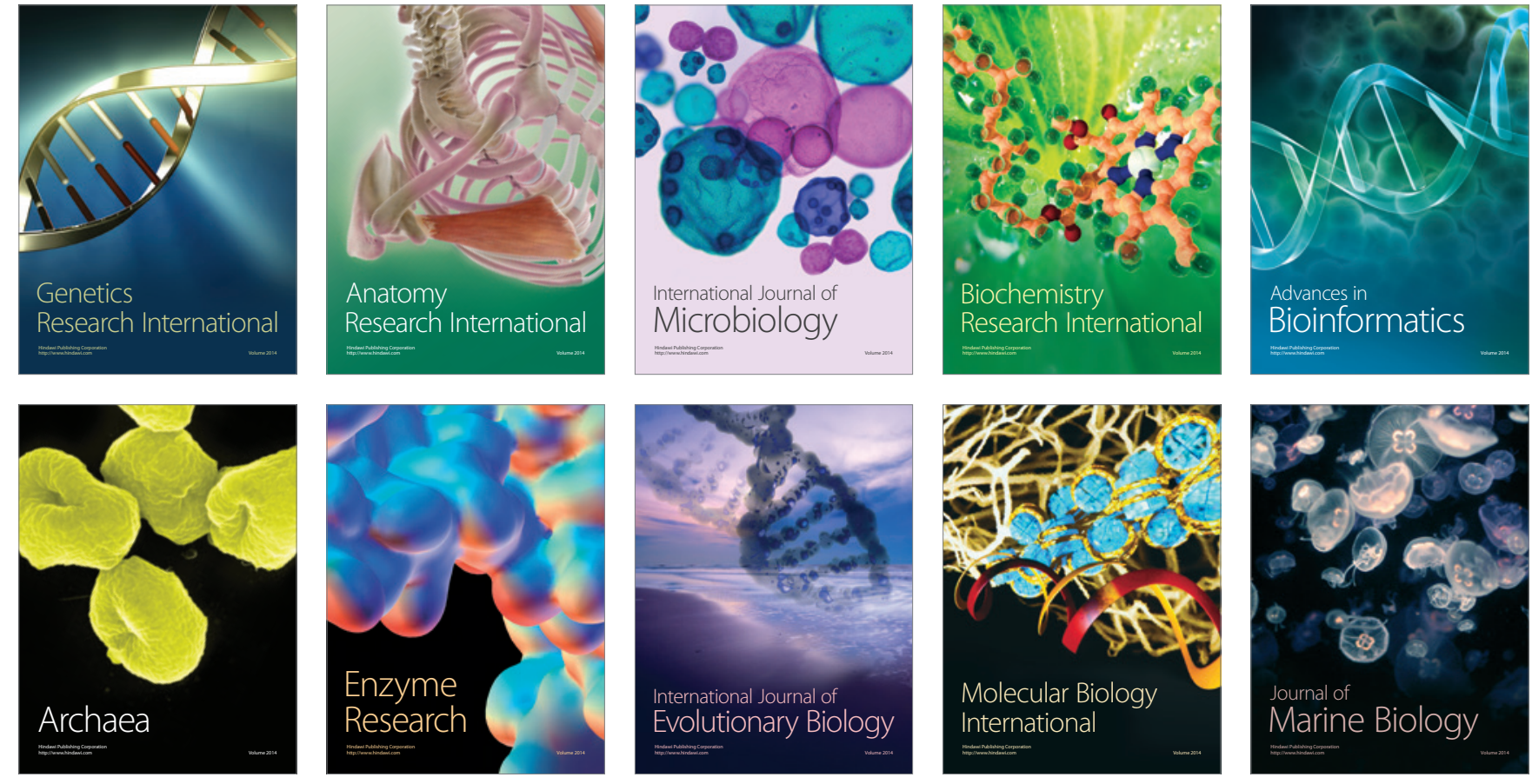